Trauma Berufskrankh 2009 · 11

[Suppl 1]:116-121

DOI 10.1007/s10039-008-1447-0

Online publiziert: 28 . November 2008

(c) Springer Medizin Verlag 2008
S. Fuchs · S. Wallstabe - U. Gerlach · C. Jürgens

BG-Unfallkrankenhaus, Hamburg

\section{Trauma des \\ Unterschenkels und des oberen Sprunggelenks}

\section{Vom einfachen Fall in die Komplikation}

Die Problematik des einfachen Falls bzw. der einfachen Fraktur sowohl am Unterschenkel als auch am oberen Sprunggelenk (OSG) wirft die Frage auf, ob es überhaupt eine einfache Fraktur gibt. Die allgemeine Erfahrung aus der Unfallchirurgie lehrt, dass es sicherlich eine einfache Fraktur am Schienbein oder am Knöchel gibt. Das Problem ist aber, zu klären, welche Fraktur wirklich einfach ist. Hierzu bedarf es einer exakten Diagnostik, Analyse und individuellen Therapie durch ein erfahrenes und qualifiziertes Team, das in der Lage und bereit ist, auch eigene Fehlleistungen oder Fehlergebnisse zu erkennen und rechtzeitig zu korrigieren. Das Endergebnis definiert für den Patienten zum großen Teil die Schwere der erlittenen Verletzung.

Dass es bei der operativen Versorgung einfacher Brüche dennoch zu teilweise schwerwiegenden Komplikationen kommen kann, soll im Folgenden an den unterschiedlichen Lokalisationen des Unterschenkels und des OSG exemplarisch erläutert werden.

\section{Tibiakopf}

Die häufigsten Ursachen für Tibiakopffrakturen sind Sturz und Verkehrsunfälle.

\section{Mögliche Komplikationen}

Aufgrund der Nähe zum Kniegelenk stellt die Tibiakopffraktur eine schwere Ver- letzung dar, die ohne adäquate Therapie ernste Folgen nach sich ziehen kann. Hierzu zählen:

- die posttraumatische Gonarthrose bei nicht anatomisch ausgeheilter Gelenkfläche,

- Achsfehlstellungen und

- unbehandelte Begleitverletzungen.

Ziel der Therapie sollte daher die Wiederherstellung der Gelenkflächen und Achsverhältnisse sein. Die Osteosynthese sollte so stabil sein, dass eine frühfunktionelle Übungsbehandlung möglich ist.

\section{Diagnose und Therapie}

Zur Diagnostik zählt neben der konventionellen Röntgendiagnostik des Kniegelenks in 2 Ebenen eine zusätzliche Computertomographie (CT) zur präzisen Darstellung des Frakturverlaufs und zur exakten Operationsplanung mit den entsprechenden Zugängen [9].

Der operative Zugang über große Schnitte (z. B. Y-Schnitt) zur ausgedehnten Freilegung des Knochens sollte vermieden werden. Gezielte Längsschnitte als anterolateraler bzw. -medialer Zugang sollten gemäß der präoperativen Planung und Gelenkpathologie (CT) gewählt werden. Bikondyläre Plattenosteosynthesen (- Abb. 1a) sollten der Vergangenheit angehören.

Auch bei komplexen Frakturen ist die unilaterale, winkelstabile Plattenosteosynthese, ggf. in Kombination mit einer Anti- gleitplatte von medial, Standard. Das Beispiel der bikondylären Plattenosteosynthese zeigt die typische Komplikation einer „heavy metal“-Osteosynthese am Tibiakopf mit Auftreten einer Osteitis und freiliegendem und avitalem Knochen zwischen den Platten sowie einem Kniegelenkempyem (- Abb. 1b). Nach ausgedehnten Sequestrektomien, bilateraler Arthrotomie und Stabilisierung im Fixateur externe ( $\bullet$ Abb. 1c) heilte der Befund nach Beruhigung der Osteitis und Aufbau mit Spongiosaplastiken sowie Lappendeckung des Hautweichteildefekts unter deutlicher Deformierung der Gelenkfläche aus (• Abb. 1d).

\section{Unterschenkel}

Die Tibiaschaftfraktur stellt eine Domäne der intramedullären Osteosynthese dar [6]. Insbesondere der unaufgebohrte Tibianagel ist nahezu zum Standardimplantat zur Versorgung von Unterschenkelfrakturen geworden [14]. Trotzdem drohen Fallstricke und Fehlermöglichkeiten bei seiner Anwendung.

\section{Mögliche Komplikationen}

Freedman u. Johnson [2] konnten eine Achsfehlstellung bei den proximalen $\mathrm{Ti}$ biafrakturen nach intramedullärer Osteosynthese von $58 \%$ nachweisen. Diese resultierten überwiegend aus einer mangelnden Reposition, insbesondere der proximalen Fraktur, falschen Nagelein- 
trittstellen und einer verbleibenden Instabilität. Vor allem die proximalen Frakturen neigen aufgrund des Muskelzugs der Hamstring- und der Patellarsehne zu einer Dorsal- und Valguskippung des proximalen Fragments. Die Dislokation wird durch Flexion des Kniegelenks, die zur Nagelung notwendig ist, verstärkt.

Die unterschiedlichen Nageleintrittspunkte am Tibiakopf bedingen bei den proximalen Frakturen die Fehlstellung. Ein zu weit nach medial liegender Nageleintritt führt zu einer Valgusstellung des Fragments, ein zu weit lateral gewählter Eintrittspunkt zu einer Varusstellung der Tibia. Der korrekte Marknageleintritt am Unterschenkel liegt in der Verlängerung der Tibiavorderkante, in der Regel in Projektion auf die laterale Eminentia bzw. gering medial davon (• Abb. 2).

Weitere klassische Fallstricke bei der Marknagelosteosynthese des Unterschenkels sind Rotationsfehler. Diese sollten entsprechend der Operationslagerung und dem präoperativ zu ermittelnden Torsionswinkels der gesunden Seite Beachtung finden. In der Literatur sind verschiedene Techniken entsprechend der Lagerung zur Vermeidung von Rotationsfehlern beschrieben, die im Weiteren nicht aufgeführt werden sollen [1]. Im Falle einer postoperativen Rotationsfehlstellung sollten frühzeitig ein CT zur Rotationsmessung des Unterschenkels im Seitenvergleich durchgeführt und eine frühzeitige Korrektur angestrebt werden [10].

Die Problemzone der distalen Tibia birgt bei der Versorgung mit einem unaufgebohrten Marknagel und hierbei mit dem klassischen unaufgebohrten Tibiamarknagel (UTN) verschiedene Probleme. Aufgrund der Abschnitte der Verriegelungsfläche und der Position können distale Fragmente häufig nicht ausreichend stabil verriegelt werden. Des Weiteren kann keine ausreichende Stabilität im metaphysären Bereich aufgrund des Designs des UTN gewährleistet werden

\section{(- Abb. 3).}

Neuere Nagelsysteme mit distaleren Verriegelungslöchern (auch in $45^{\circ}$-Richtung) können eine höhere Stabilität gewährleisten. Wesentliche Voraussetzung ist die richtige Reposition des distalen Fragments der Tibia. Insbesondere bei kurzen Fragmenten können Joy-Sticks als indirekte Repositionshilfen genutzt werden [12].

Zur Vermeidung einer Varisierung oder Valgisierung kann eine PollerSchraube entsprechend der Fehlstellung von ventral eingebracht werden [7]. So können beispielsweise medial vom Nagel platzierte Poller-Schrauben einer Varustendenz, lateral davon eingebrachte Schrauben einer Valgustendenz entgegenwirken.

Eine auf gleicher Höhe der Tibiafraktur vorliegende Fibulafraktur bedingt eine erhöhte Instabilität, sodass mit einer zusätzlichen Plattenosteosynthese ein deutlicher Stabilitätsgewinn erzielt werden kann [5]. Bei sehr weit distal gelegenen Frakturen und Frakturausläufern muss gleichzeitig der Einsatz alternativer Osteosyntheseverfahren überdacht werden. Die Nutzung winkelstabiler Plattensysteme in Verbindung mit minimalinvasiven Operationstechniken kann als Alternative angedacht werden (• Abb. 4, [4]).

\section{Kompartmentsyndrom}

Es stellt eine der typischen Komplikationen bei Verletzungen im Bereich des Unterschenkels dar [3]. Dabei weisen Schaftfrakturen das höchste Risiko auf, gefolgt von proximalen/distalen Tibiafrakturen.

Das in 1 Abb. 5 dargestellte Beispiel zeigt die Problematik einer internen Osteosynthese bei Kompartmentsyndrom und zweitgradig offener Unterschenkelfraktur. Dabei wurde nach primärer Stabilisierung im Fixateur externe ( $\mathbf{\square}$ Abb. 5a) frühzeitig bei offenen Kompartments eine interne Plattenosteosynthese (- Abb. 5b) von lateral durchgeführt, die letztlich zu einer Segmentresektion von $18 \mathrm{~cm}$ bei avitalen und infizierten Knochen führte (- Abb. 5c). Dieser Fall zeigt sehr deutlich einen komplikationsträchtigen Verlauf nach einem anfänglich begrenzten Problem. Aus der offenen Unterschenkelfraktur mit Kompartmentsyndrom resultierten ein Knochendefekt von $18 \mathrm{~cm}$ nach interner Osteosynthese, eine Tragzeit des Ilisarov-Ringfixateurs von 500 Tagen (- Abb. 5d), eine Dauer des Heilverfahrens von 2,5 Jahren und unabhängig von den Nebendiagnosen eine deutliche MdE in rentenberechtigter Höhe. Aufgrund eigener Erfahrung können wir uns
Trauma Berufskrankh 2009 · 11

[Suppl 1]:116-121

DOI 10.1007/s10039-008-1447-0

C) Springer Medizin Verlag 2008

S. Fuchs · S. Wallstabe - U. Gerlach .

C. Jürgens

Trauma des Unterschenkels

und des oberen Sprunggelenks. Vom einfachen Fall in die Komplikation

\section{Zusammenfassung}

Verletzungen des Unterschenkels und des oberen Sprunggelenks (OSG) müssen insbesondere in Hinsicht auf das Outcome einer exakten Diagnostik und Analyse unterzogen werden. Sie bilden die Grundlage für die entsprechende Therapie und somit die Vermeidung von Komplikationen. Bei der Versorgung sind der richtige Zugangsweg, die Wahl des richtigen Implantats und die richtige Platzierung unbedingte Voraussetzung zur Minimierung von Problemen. Ebenfalls entscheidend sind beim Eintritt von Komplikationen die Kompetenz und die Bereitschaft, diese zu erkennen und zu therapieren.

\section{Schlüsselwörter}

Vermeintlich einfacher Fall · Tibiakopffraktur - Unterschenkelschaftfraktur . Kompartmentsyndrom · OSG-Fraktur

\section{Injury to the lower leg and ankle joint. From a simple case to complications}

\section{Abstract}

Injuries to the lower leg and ankle joint need to be accurately analyzed preoperatively in regard to the outcome. This is the basis of appropriate therapy and therefore the avoidance of complications. Correct access, the right choice of implant and correct placement are prerequisites for minimizing difficulties. When complications do occur, it is crucial to have the competence and readiness to recognize and treat them

\section{Keywords}

Supposedly simple case - Tibial head fracture . Lower leg fracture - Compartment syndrome. Ankle joint fracture 

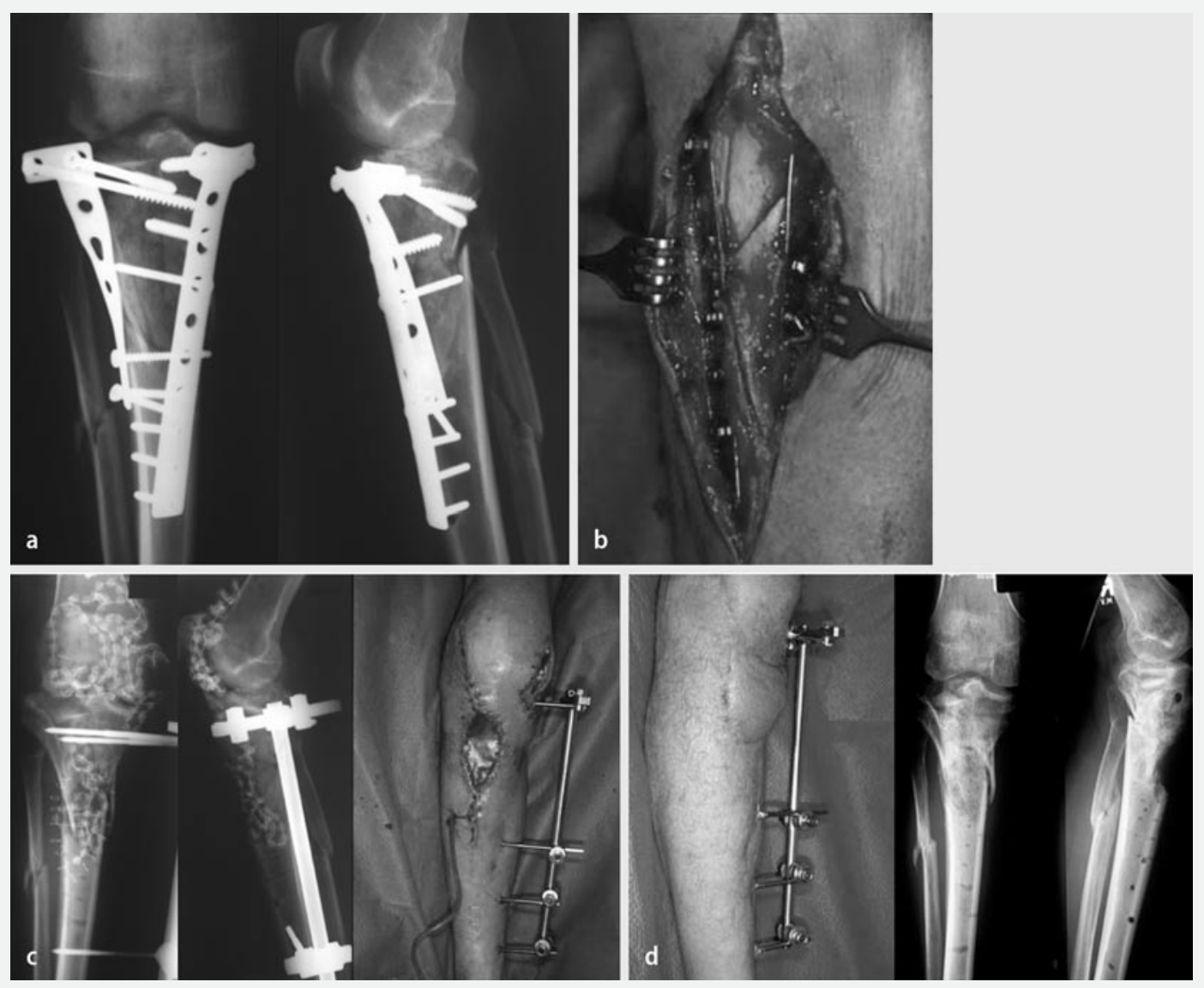

Abb. $1 \varangle$ Tibiakopf, a bilaterale Plattenosteosynthese, $\mathbf{b}$ Osteitis bei Doppelplattenosteosynthese, c Stabilisierung im Fixateur externe nach Materialentfernung, Sequestrektomie und bilateraler Arthrotomie mit Septopalketteneinlage und Defektdeckung mit Epigard, d Ausheilungsergebnis nach lappenplastischer Deckung und Spongiosaplastik

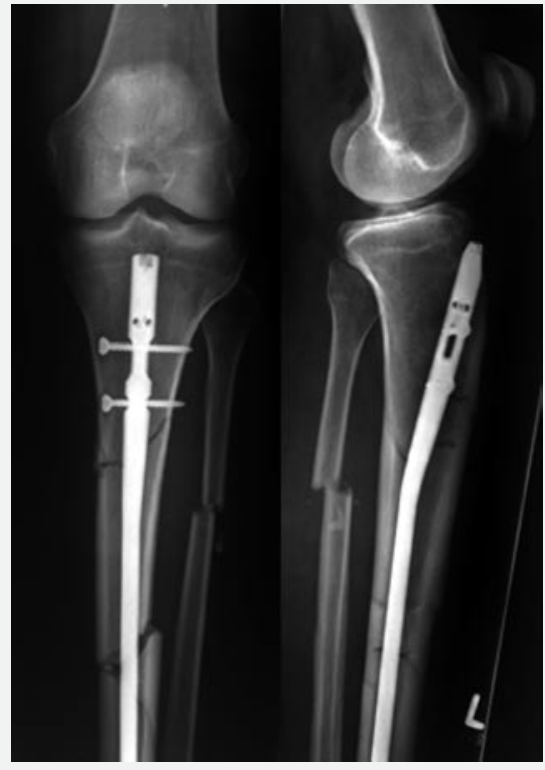

Abb. $2 \Delta$ Tibiakopf, korrekte Nageleintrittsstelle

Abb. 3 D Distale Tibia, Fehlstellung bei unzureichender Stabilität durch UTN

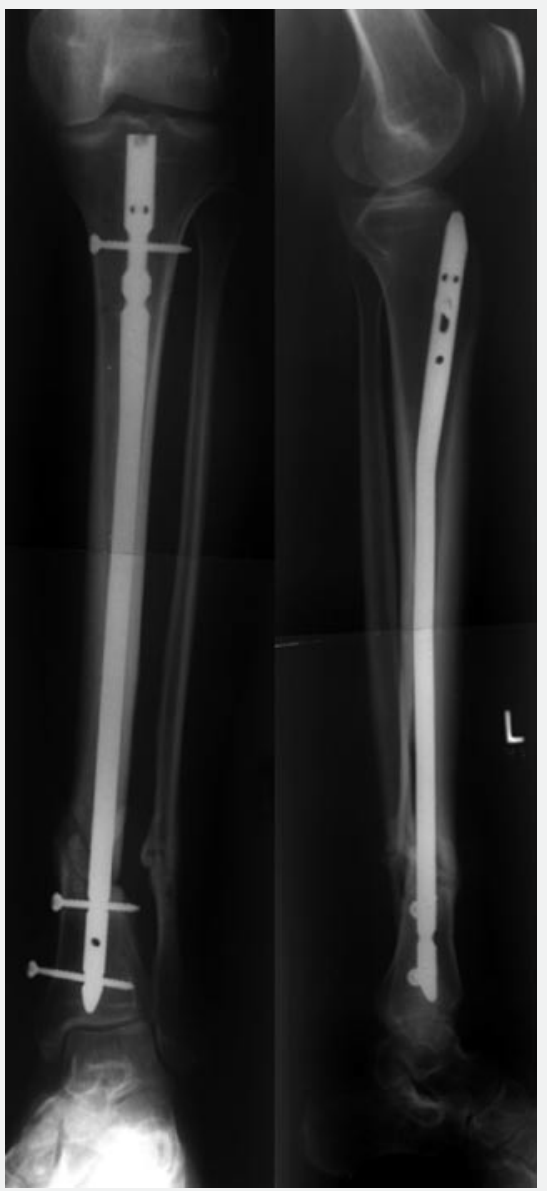

Abb. $4 \Delta$ Distale Tibia, Pilon-TiFix ${ }^{\oplus}$ 
Abb. 5 a Zweitgradig offene Unterschenkelfraktur mit Kompartmentsyndrom und Stabilisierung im Fixateur externe, b Umstieg auf interne Plattenosteosynthese bei Kompartmentsyndrom mit Frühinfekt, c Segmentresektion und Stabilisierung im Ilisarov-Ringfixateur, d Segmenttransport im llisarovRingfixateur
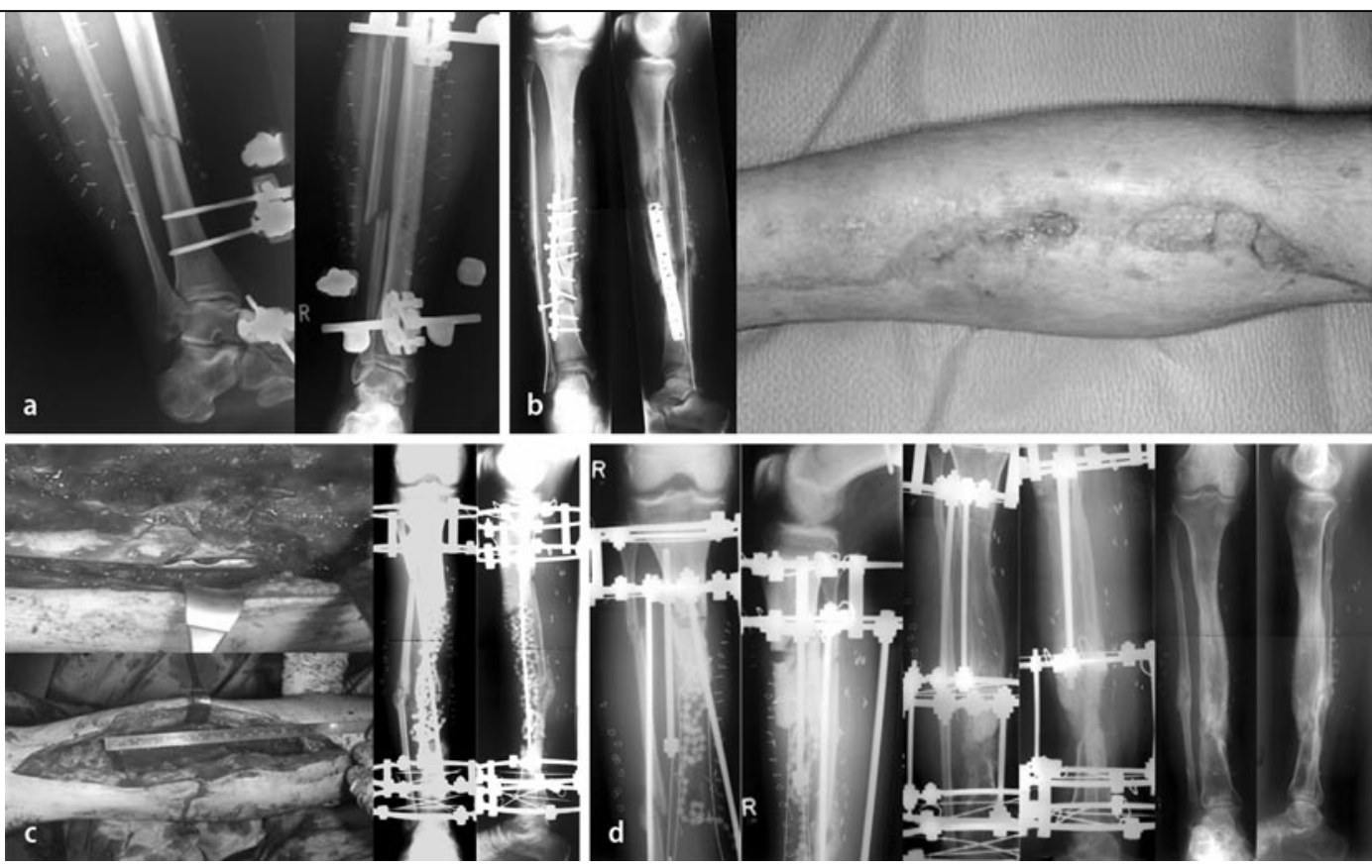

dem von Friedel [3] dargelegten Behandlungskonzept, auch bei zweit- und drittgradig offenen Frakturen sowie Kompartmentsyndrom auf interne Osteosynthesen zu wechseln, nicht anschließen.

\section{Oberes Sprunggelenk}

Verletzungen des OSG stellen mit einer Inzidenz im europäischen Raum von 1:10.0oo Einwohnern einen erheblichen Anteil des Patientenguts unfallchirurgischer Kliniken dar [8]. Dennoch können bei der Diagnostik und der Behandlung der vermeintlich einfachen Knöchelfraktur erhebliche Probleme auftreten.

\section{Diagnostik}

Neben der Anamneseerhebung zur Analyse des Unfallmechanismus stellt die Röntgenuntersuchung in 2 Ebenen sowie in $30^{\circ}$ Innenrotation die Standarddiagnostik dar. Impression der distalen Tibiagelenkfläche oder ein vorderes oder hinteres Tibiakantenfragment können auf konventionellen Röntgenaufnahmen verborgen bleiben, sodass in vielen Fällen nur die CT das gesamte Verletzungsausmaß darstellt und den Weg für eine sichere Therapieentscheidung ebnet.

\section{Therapie}

Die Länge, die Rotation und die Achse der Fibula müssen exakt wiederhergestellt werden. Zusätzlich sind die häufig ebenfalls auftretenden Außenrotations-, Adund Abduktionsfehlstellungen zu vermeiden [13]. Intraoperativ schließt sich die Überprüfung der Stabilität des Syndesmosenkomplexes an. Bewährt haben sich der Druck von ventral auf den Außenknöchel zur Überprüfung der vorderen Anteile und der lateral gerichtete Zug mit dem Einzinkerhaken zur Inspektion der interossären Anteile [11]. Bei Syndesmoseninstabilität hat sich die Stellschraube als Goldstandard erwiesen [11]. Hierbei muss Wert darauf gelegt werden, die Schraube parallel zur Rotationsebene des OSG, also um $30^{\circ}$ aus der frontalen Ebene von dorsolateral nach anteromedial ansteigen zu lassen. Die intraoperative Überprüfung der Frakturreposition gelingt in der Durchleuchtung in 3 Ebenen. Die sichere Beurteilung der Torsion und der Stellung der Fibula in der Inzisur ist nur im postoperativen CT möglich, sodass bei Verdacht auf Fehlstellung eine Computertomographie zu fordern ist. Eine Korrektur sollte grundsätzlich so früh wie möglich erfolgen. Eine Spätrekonstruktion einer übersehenden Syndesmosenverletzung mit nachfolgender OSGInkongruenz weist bislang schlechtere Ergebnisse auf.

\section{Fallbeispiel}

- Abb. 6 zeigt das Bild einer auswärts versorgten OSG-Fraktur bei einer 51-jährigen Patientin mit Nebendiagnosen einer chronischen Polyarthritis, Kortisondauermedikation sowie einem fraglichen Alkoholabusus. Nach Materialentfernung fand sich sowohl nativradiologisch als auch computertomographisch eine weite Syndesmose mit Inkongruenz des OSG. Gleichzeitig bestand eine Mitverletzung des Pilon tibiale, welche bis dahin nicht bekannt war. Bei Beschwerdesymptomatik wurde der Entschluss zur Syndesmosenrekonstruktion mit temporärer Stabilisierung mit 2 Stellschrauben gefasst (- Abb. 6c). Nach Materialentfernung (- Abb. 6d) kam es im Verlauf zu chronischen Beschwerden mit Belastungsinsuffizienz und Bewegungseinschränkung des OSG, weswegen eine OSGProthese Modell Salto implantiert wurde (- Abb. 6e). Diese musste nach 1 Jahr wegen septischer Lockerung explantiert werden, es folgten Stabilisierung im AORahmen-Fixateur sowie eine Spongiosaplastik (• Abb. 6f). Die nach Entfernung des Rahmenfixateurs instabile Arthrodesensituation wurde mit Arthrodesennagel bei fehlenden Infektzeichen behandelt (- Abb. 6g). Nach 6 Monaten kam es zur Infektion des einliegenden Arthrodesennagels mit Hautweichteildefekt, sodass eine Rearthrodese im Ilisarov-Ring- 


\section{Der vermeintlich einfache Fall}
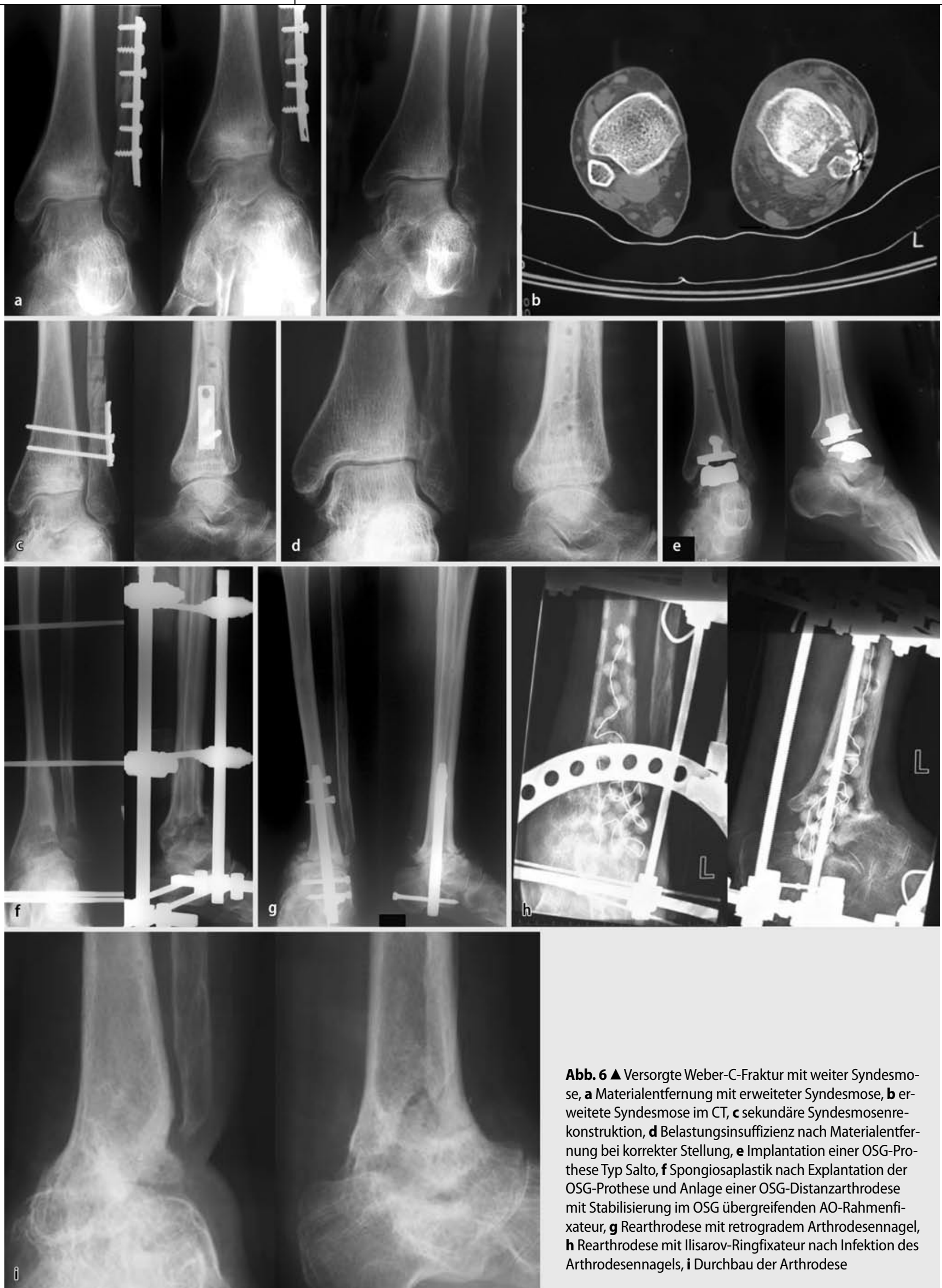

Abb. $6 \Delta$ Versorgte Weber-C-Fraktur mit weiter Syndesmose, a Materialentfernung mit erweiteter Syndesmose, b erweitete Syndesmose im CT, c sekundäre Syndesmosenrekonstruktion, d Belastungsinsuffizienz nach Materialentfernung bei korrekter Stellung, e Implantation einer OSG-Prothese Typ Salto, $\mathbf{f}$ Spongiosaplastik nach Explantation der OSG-Prothese und Anlage einer OSG-Distanzarthrodese mit Stabilisierung im OSG übergreifenden AO-Rahmenfixateur, $\mathbf{g}$ Rearthrodese mit retrogradem Arthrodesennagel, h Rearthrodese mit llisarov-Ringfixateur nach Infektion des Arthrodesennagels, i Durchbau der Arthrodese 
fixateur (• Abb. 6h) mit Sequestrektomie des Arthrodesennagels erforderlich wurde. Nach 4 Monaten konnte der Ringfixateur bei verheilter Arthrodese entfernt werden (• Abb. 6i).

\section{Fazit}

\section{Zum Erkennen der einfachen Fraktur am Unterschenkel und OSG bedarf es ne- ben der konventionellen Röntgendiag- nostik häufig zusätzlich einer CT-Unter- suchung, welche zugleich der präopera- tiven Planung in Bezug auf den Zugang sowie der Osteosyntheseform dient. Die dargestellten verletzungstypischen Fall- stricke können Komplikationen redu- zieren, sodass die vermeintlich einfache Fraktur auch unproblematisch behandelt werden kann.}

\section{Korrespondenzadresse}

\section{Dr. S. Fuchs}

BG-Unfallkrankenhaus Hamburg, Bergedorfer Straße 10, 21033 Hamburg S.Fuchs@buk-hamburg.de

Interessenkonflikt. Der korrespondierende Autor gibt an, dass kein Interessenkonflikt besteht.

\section{Literatur}

1. Clementz BG (1989) Assessment of tibial torsion and rotational deformity with a new fluoroscope technique. Clin Orthop 245: 199-209

2. Freedmann EL, Johnson EE (1995) Radiographic analysis of tibial fracture malalignment following intramedullary nailing. Clin Orthop 315: 25

3. Friedel W (2001) Diagnostik und Therapie des Kompartmentsyndroms des Unterschenkels. Trauma Berufskrankh [Suppl 2] 3: S163-S166

4. Fuchs S, Wolter D, Kranz HW et al. (2001) Titan-Fixateur-interne-Systeme mit multidirektionaler Winkelstabilität im Unterschenkel- und Fußbereich. Trauma Berufskrankh [Suppl 4] 3: S425-S428

5. Höntzsch D, Weller S, Dürselen L et al. (1993) Die begleitende Fibulaosteosynthese bei der kompletten Unterschenkelfraktur. Traumatologie aktuell, Bd 9. Thieme, Stuttgart New York

6. Kappus M, Börner M (2001) Marknagelung gebohrt versus ungebohrt. Trauma Berufskrankh [Suppl 2] 3: S125-S129

7. Krettek C, Schandelmaie P, Tscherne H (1997) Neue Entwicklungen bei der Stabilisierung dia- und metaphysärer Frakturen der langen Röhrenknochen. Orthopäde 26: 408-421

8. Ochs U, Winter E, Weise K (2001) Malleolenfrak turen. Trauma Berufskrankh 3: 338-343

9. Petersen W, Zantop T, Raschke M (2006) Tibiakopffraktur. Unfallchirurg 109: 219-234

10. Pröbstel M, Richter FJ, Börner M (1999) Ist die routinemäßige postoperative CT-Messung nach Marknagelung von Ober- und Unterschenkelfrakturen indiziert? Trauma Berufskrankh 1: 152-157
11. Recum J von, Mayer H, Wendl K et al. (2006) Frische Verletzung des OSG. Trauma Berufskrankh [Suppl 1] 8: S14-S19

12. Stedtfeld H-W (2001) Marknagelfixation distaler Tibiafrakturen. Trauma Berufskrankh [Suppl 2] 3: S150-S155

13. Wernicke F, Otto W (2003) Knöchelbruch - eine einfache Fraktur? Trauma Berufskrankh 5: 149-155

14. Zwipp H, Grass R, Bachmann L et al. (2001) UTN Anwendung und Fehlermöglichkeiten. Trauma Berufskrankh [Suppl 2] 3: S130-S134 